\title{
Novel Approach for MRI Image Quality Analysis by Hybrid Preprocessing Techniques
}

\author{
K Phaneendra ${ }^{1}$ | B Tirupathi Rao ${ }^{2}$ | B Nikhila Sree ${ }^{2}$ J Haswanth Kumar ${ }^{2}$ | B Harshavardhan Raju ${ }^{2}$ \\ ${ }^{1}$ Assistant Professor, Department of Electronics and Communication Engineering, Raghu Institute of Technology, \\ Visakhapatnam, India. \\ ${ }^{2}$ UG Student, Department of Electronics and Communication Engineering, Raghu Institute of Technology, Visakhapatnam, \\ India.
}

\section{To Cite this Article}

K Phaneendra, B Tirupathi Rao, B Nikhila Sree, J Haswanth Kumar and B Harshavardhan Raju, "Novel Approach for MRI Image Quality Analysis by Hybrid Preprocessing Techniques", International Journal for Modern Trends in Science and Technology, Vol. 06, Issue 05, May 2020, pp.:27-32; https://doi.org/10.46501/IJMTST060505

\section{Article Info}

Received on 24-March-2020, Revised on 20-April-2020, Accepted on 25-April-2020, Published on 29-April-2020.

\section{ABSTRACT}

Image pre-preparing methods are utilized to improve the nature of a image before handling into an application. This uses a little neighborhood of a pixel in an information image to get another splendor esteem in the yield image. These pre-preparing methods are likewise called as filtration and goals upgrade. The clinical image quality parameters are fundamentally clamor and goals. The fundamental goal of this paper is to improve the image quality by denoising and goals upgrade. A large portion of the imaging methods are corrupted by clamor. So as to protect the edges and form data of the clinical images, the effective denoising and an improved upgrade method is required. This paper focuses the normal, middle and wiener sifting for image denoising and an addition based Discrete Wavelet Transform (DWT) strategy for goals improvement. The presentation of these strategies is assessed utilizing Peak Signal to Noise Ratio (PSNR). From the outcomes, it uncovers that the effective denoising and goals upgrade method is basic for image pre-handling.

KEYWORDS: Image preprocessing, Noise, Denoising, Discrete Wavelet Transform, Peak Signal to Noise Ratio.

Copyright ( (2014-2020 International Journal for Modern Trends in Science and Technology. All rights reserved. DOI: https://doi.org/10.46501/IJMTST0605005

\section{INTRODUCTION}

The Magnetic Resonance Imaging (MRI) is to see the inward structures of the body in detail particularly for imaging delicate tissues and it doesn't utilize any radiations. Mind tumor is an anomalous development of tissues in the cerebrum and is fundamentally brought about by radiation to the head, hereditary hazard, HIV disease, cigarette smoking and furthermore because of ecological poisons. Serious issue in image division is wrong determination of the tumor district which gets diminished predominantly because of the differentiation, obscure, clamor, antiquities, and bending.

No exact discovery of tumor district because of the nearness of clamor in MR image. Indeed, even limited quantity of clamor can change the arrangement. Dim issue is comprised of neuronal cell bodies. The Gray issue incorporates locales of the cerebrum associated with muscle control, tangible recognition, for example, seeing and hearing, memory, feelings, and discourse. White 
issue is one of the two parts of the focal sensory system and comprises for the most part of glial cells and myelinated axons that transmit signals starting with one locale of the cerebrum then onto the next and between the cerebrum and lower mind focuses. Uproarious image can cause misclassifications of Gray Matter (GM) as White Matter (WM). So the clamor is preprocessed utilizing denoising strategy. Goals of a image is constantly an issue in clinical image handling which implies loss of value at the image edges. Goals upgrade is utilized to safeguard the edges and form data. The significant utilization of these methods is location of tumor cells in human body $[1,2]$.

Improving the denoising alongside the edges isn't performed so well in this strategy [3]. So as to altogether quicken the calculation, the channels are acquainted with dispense with irrelevant neighborhoods from the weighted normal used to denoise each image pixel. These channels depend on normal dark qualities just as angles, pre-grouping neighborhoods and accordingly decreasing the quadratic multifaceted nature to a straight one and reducing the impact of less-related regions in the denoising of a given pixel. Some portion of the progressing endeavors incorporates the examination of image qualities that give great setting characterizations to image denoising [4]. In spite of the fact that the backwards channel functions admirably when no clamor is available, the Wiener channel performs much better and is progressively adaptable. In any case, the Wiener channel expect information on the debasement work and the force spectra of both the commotion and the first image. Most image rebuilding strategies require some information on the debasement work, however the Wiener channel specifically presents the extra trouble of knowing the force spectra, the clamor power range can be viably evaluated by examining a moderately uniform area of enthusiasm for the corrupted image. Be that as it may, acquiring the range of the first sign is increasingly troublesome. This prerequisite makes the Wiener channel less helpful in numerous down to earth applications. Besides, the Wiener channel gives a sound hypothetical establishment whereupon other rebuilding strategies [5]. Low and high recurrence data is viably separated by utilizing Haar wavelet change however commotions in the low recurrence sub-band are smoothened and in the high recurrence sub-groups are honed by utilizing the smooth PWL (Piece Wise Linear) channel and another PWL channel individually has an extremely palatable clamor evacuating property just as improves the visual nature of images that contain low difference. The exhibition of both wavelet change and PWL has been looked at [6]. The image goals improvement strategy utilizing Discrete Wavelet Transform (DWT) is giving preferable outcomes over some other procedure [7].

\section{DENOISING MECHANISM}

The vast majority of the imaging methods are debased by commotion with the goal that the image is preprocessed utilizing denoising strategy to separate the helpful data. To dissect the clinical image must be expelled from the MRI image for holding the first data. Clamor in clinical imaging is mostly brought about by variety in the locator affectability, decreased article visibility(low differentiate), concoction or photographic impediments, and irregular vacillations in radiation signal.

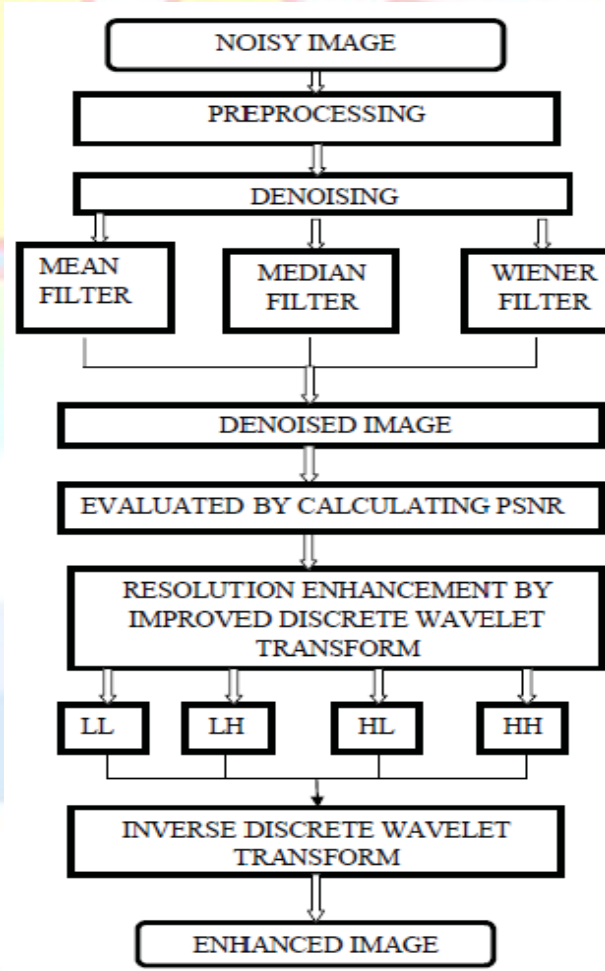

Fig. 1 Overview of the proposed work i.e sectioning the mind tissues, at first the clamor

\section{A. Gaussian noise}

From the outset the MRI image is taken as a data. The MRI image is incorporated with Gaussian uproar. The denoising is performed using averaging channel, center channel and wiener channel. The introduction of these denoising strategies is assessed using Peak Signal to Noise Ratio. Fig .1 shows the layout of the proposed 
work. Image commotion is characterized as the mark appointed at first at any pixel and the pixel course of action will be in an irregular likelihood thickness work equivalent to that of ordinary appropriation, additionally called Gaussian circulation. The mean (normal) and change (standard deviation) are the characterizing factors. Gaussian clamor whose recurrence range after a Fourier change has a chime molded bend and is symmetric around mean. So as to test the opposition of a image and furthermore to assess the presentation of the MRI cerebrum image Gaussian clamor is included and sifted utilizing some commotion channels. Every pixel in the uproarious image contains both genuine pixel esteem what's more, irregular Gaussian dissemination clamor esteem. Gaussian circulation condition is given underneath.

$$
f(x)=\frac{1}{\sigma \sqrt{2 \pi}} \cdot e^{\frac{-1}{2}\left(\frac{x-\mu}{\sigma}\right) 2}
$$

where $\mu$ signifies mean (normal) estimation of $\mathrm{x}$ and $\mathrm{o}$ indicates standard deviation of $\mathrm{x}$.

\section{B. Averaging Filter}

Mean channel or averaging channel is a basic direct channel and simple usage technique for smoothing images. Normal channel is frequently used to decrease clamor and furthermore diminish the measure of force variety starting with one pixel then onto the next. Here, first take a normal that is aggregate of the components and partition the whole by the quantity of components. Next, supplant every pixel in a image by the normal of pixels in a square window encompassing this pixel $[8,9,10]$. Fig 2 portrays the usefulness behind the averaging channel.

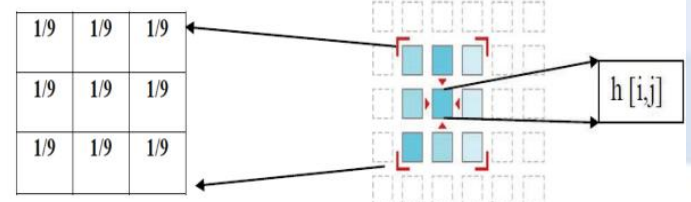

Fig. 2 Functionality behind the averaging filter

$$
h[i, j]=\frac{1}{M} \sum_{(k, l) \in N} f(k \cdot l)
$$

where $\mathrm{M}$ is the all out number of pixels in the local $\mathrm{N}$ and $\mathrm{k}, 1=1,2$.. For instance, a $3 \mathrm{X} 3$ neighborhood about $[i, j]$ yields:

$$
h[i, j]=\frac{1}{9} \sum_{k=i-1}^{i+1} \sum_{j=i-1}^{i+1} f(k . l)
$$

Issue with averaging of channel is that it can evacuate commotion all the more adequately in bigger windows, yet in addition obscure the subtleties in a image.

\section{Median Filter}

Middle sifting is a nonlinear activity regularly utilized in image preparing to decrease commotion. A middle channel is more successful than convolution when the objective is to all the while lessen commotion and protect edges. The middle channel additionally like mean channel that thinks about every pixel in the image thus and takes a gander at its close by neighbors to choose whether or not it is the delegate of its environmental factors. Rather than just supplanting the pixel esteem with the mean of neighboring pixel esteems, it replaces it with the middle of those qualities. The middle is determined by arranging all the pixel esteems from the encompassing neighborhood into numerical request and afterward supplanting the pixel being considered with the center pixel esteem $[11,12]$. Note that on the off chance that the window has an odd number of sections, at that point the middle is easy to characterize. It is the center an incentive after all the sections in the window are arranged numerically. For a considerably number of passages, there is more than one potential middle. In middle separating, the neighboring pixels are positioned by brilliance (force) and the middle worth turns into the new incentive for the focal pixel. Fig 3 shows the working guideline of middle channel.

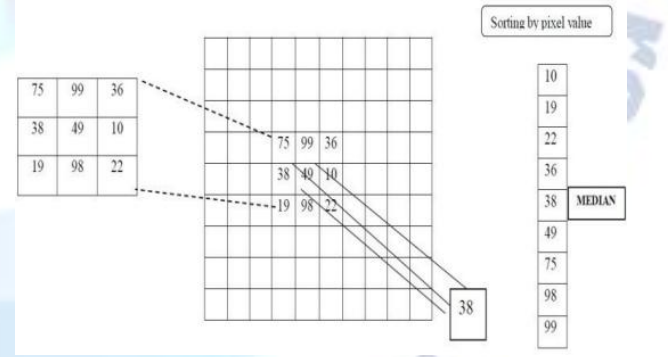

Fig .3 Working principle of median filter

Favorable circumstances of middle channel are there is no decrease interestingly across ventures, since yield esteems accessible comprise just of those present in the area (no midpoints). The middle is less delicate than the intend to extraordinary qualities (anomalies), those outrageous qualities are all the more adequately expelled. The disservice of middle channel is now and then this isn't abstractly acceptable at managing huge measure of Gaussian clamor as the mean channel.

\section{Wiener Filter}


The significant utilization of wiener channel is to diminish the measure of clamor present in a image by examination with an estimation of the ideal quiet sign. It depends on a measurable methodology. Wiener channels are portrayed by three significant variables. 1) Assumption: The fixed straight stochastic procedures of image and clamor with known otherworldly qualities or known autocorrelation and cross-connection 2) Requirement: the channel must be genuinely feasible/causal 3) Performance rule: least mean-square mistake (MMSE).

This channel is as often as possible utilized during the time spent deconvolution. The backwards sifting is a reclamation method for deconvolution, i.e., when the image is obscured by a realized low pass channel, it is conceivable to recoup the image reverse separating or summed up opposite sifting. Nonetheless, reverse sifting is delicate to added substance commotion. The methodology of diminishing debasement at a time prompts to build up a rebuilding calculation. The Wiener sifting executes an ideal tradeoff between backwards separating and commotion smoothing $[13,14,15]$. It expels the added substance clamor and transforms the obscuring all the while. The Wiener separating is ideal as far as the mean square mistake. As it were, it limits the general mean square mistake during the time spent opposite separating and clamor smoothing. The Wiener sifting is a straight estimation of the first image. The symmetry standard suggests that the Wiener channel in Fourier area can be communicated as follows:

$$
W(f 1, f 2)=\frac{H^{*}\left(f_{1}, f_{2}\right) S_{x x}\left(f_{1}, f_{2}\right)}{\left|H\left(f_{1}, f_{2}\right)\right|^{2} S_{x x}\left(f_{1}, f_{2}\right)+S_{\eta \eta}\left(f_{1}, f_{2}\right)}
$$

where $\operatorname{Sxx}(\mathrm{f} 1, \mathrm{f} 2), \operatorname{Syy}(\mathrm{f} 1, \mathrm{f} 2)$ are power spectra of the first image and the added substance clamor, and $\mathrm{H}$ (f1, f2) is the obscuring channel. It is anything but difficult to see that the Wiener channel has two separate parts, an opposite sifting part and a clamor smoothing part. It isn't just plays out the deconvolution by backwards sifting (high pass separating) yet in addition expels the clamor with a pressure activity (low pass separating).

\section{RESOLUTION ENHANCEMENT}

Goals of a image is constantly an issue in clinical image handling. Goals is a proportion of the measure of detail data in the image. High goals gives more image subtleties. At first the image is preprocessed utilizing denoising. In the wake of denoising it brings about commotion decrease and loss of value at the image edges. Goals improvement is utilized to save the edges and shape data of a sifted image. So as to fragment a image precisely protecting the edges and shape data is significant.

Goals is the estimation of nature of a denoised image. So as to upgrade the goals of a image an improved discrete wavelet change is proposed. The improved DWT jelly the edges and the form data. The exhibition of goals upgrade method is estimated utilizing Peak Signal to Noise Ratio.

\section{A. Discrete Wavelet Transform}

Wavelets are assuming a critical job in many image handling applications. A wavelet change (WT) depends on wavelets. It is utilized to break down a sign (image) into various recurrence segments at various goals scales (for example multiresolution). This permits uncovering image's spatial and recurrence characteristics at the same time. Any wavelet-based image preparing approach has the accompanying advances. Register the 2D-DWT of a image, change the change coefficients (for example sub-groups), and register the opposite change.

Wavelet changes are utilized in a wide scope of image preparing applications, for example, image and video pressure, highlight identification and acknowledgment, and image denoising. The 2-D wavelet disintegration of a image is performed by applying the 1-D discrete wavelet change (DWT) along the lines of the image first, and afterward the outcomes are deteriorated along the segments [16 ,17, 18]. One level DWT (with Daubechies $9 / 7$ as wavelet work) is utilized to deteriorate an information image into various sub-band images. Three high recurrence sub-groups (LH, HL, and $\mathrm{HH})$ contain the high recurrence parts of the information image. The sub-band images are alluded to low-low (LL), low-high (LH), high-low (HL) and high-high (HH). The recurrence segments of those four sub-groups are inserted to cover the full recurrence range of the first image. The addition strategy is utilized to build the quantity of pixels in a image. The high recurrence sub-band of the image is added to low recurrence sub-groups of the image to give high goals improved image. Fig 4 and Fig 5 shows the one level decay of DWT and square graph of DWT. 


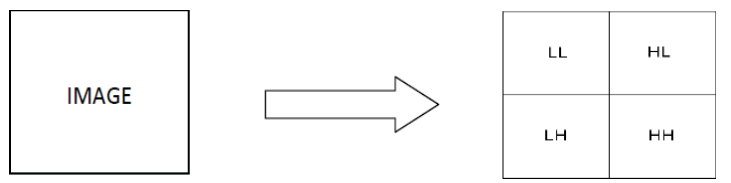

Fig .4 One level decomposition

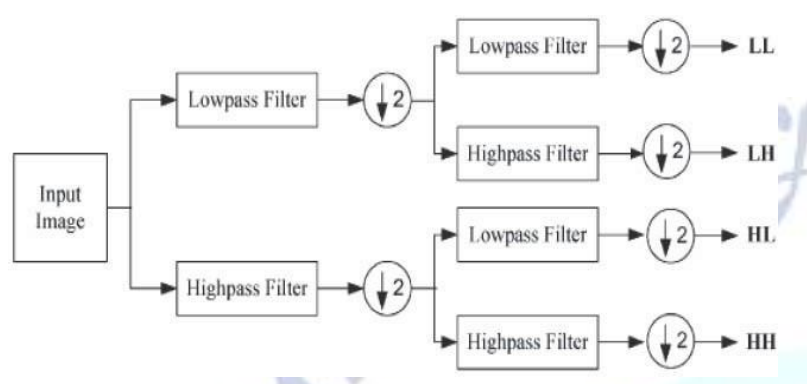

Fig. 5 Block Diagram of Discrete Wavelet Transform

The low goals image (LL sub-band), without quantization (i.e., with twofold exactness pixel esteems) is utilized as the contribution for the proposed goals upgrade process. At the end of the day, low recurrence sub-band images are the low goals of the first image. In this way, rather than utilizing low-recurrence sub-band images, which contains less data than the first information image, the information image is utilized through the obliteration procedure $[19,20]$. Subsequently, the info low-goals image is deteriorated with the half of the devastation factor to improve DWT.

\section{$B$. Inverse Discrete Wavelet Transform}

A procedure by which parts can be collected go into the first image without loss of data is called reproduction. Converse Discrete Wavelet Transform (IDWT) remakes a image from the estimate and detail coefficients got from decay. The exhibition of denoised and improved image is assessed by ascertaining PSNR esteem.

\section{QUALITY ANALYSIS}

The nature of the preprocessed images is dissected utilizing Peak Signal to Noise Ratio (PSNR). It is characterized as the proportion between the greatest conceivable intensity of a image and the intensity of undermining clamor proportion of the pinnacle mistake. Pinnacle signal-to-commotion proportion is estimated in decibels between two images. This proportion is frequently utilized as a quality estimation between the first and a denoised image. To process the PSNR, first, compute the mean-squared blunder. Mean Square Error (MSE) is the aggregate squared mistake between the denoised and the first image.

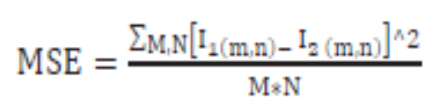

where $I 1(m, n)$ means unique image, $I 2(m, n)$ indicates denoised image and $\mathrm{M}$ and $\mathrm{N}$ are the quantity of lines and segments in the info images

At that point it tends to be anything but difficult to register PSNR utilizing the accompanying condition:

$$
\text { PSNR }=10 \log _{10}\left(\frac{\mathrm{R}^{2}}{\mathrm{MSE}}\right)
$$

where, $\mathrm{R}$ is the most extreme variance in the info image information type. For instance, in the event that the information image has a twofold exactness coasting point information type, at that point $\mathrm{R}$ is 1 . In the event that it has a 8 -piece unsigned whole number information type, $\mathrm{R}$ is 255 , and so on. Consistently, if the PSNR is higher it gives the better nature of the reproduced image.

\section{EXPERIMENTAL RESULTS}

The boisterous image is taken as the info image and denoising is performed utilizing normal, middle and wiener channel. Fig 6 and Fig 7 show the information image and denoised images and Fig 8 shows execution of the denoised image

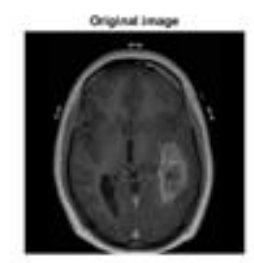

Fig.6 Input image
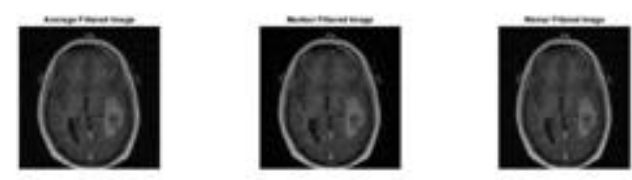

Fig. 7 Denoised images a) Averaging filter b) Median filter c) Wiener filter 


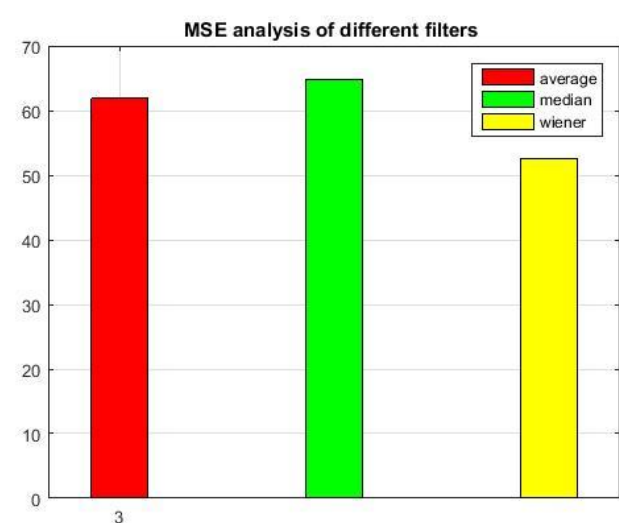

Fig 8 Performance of the denoised image

The denoised image is deteriorated into four sub-groups (LL, LH, HL, HH) utilizing addition based DWT. Fig 9 shows the disintegration levels of denoised image. Fig 10 delineates the denoised and goals upgraded image. From the figure, it shows that outwardly and scientifically goals upgraded image gives better quality for preparing a image into various applications. Fig 11 shows the exhibition examination of denoised and the goals upgraded image. The PSNR estimation of the image is improved from $30 \mathrm{~dB}$ to $38 \mathrm{~dB}$ in the goals upgraded image and blunder likewise diminished.
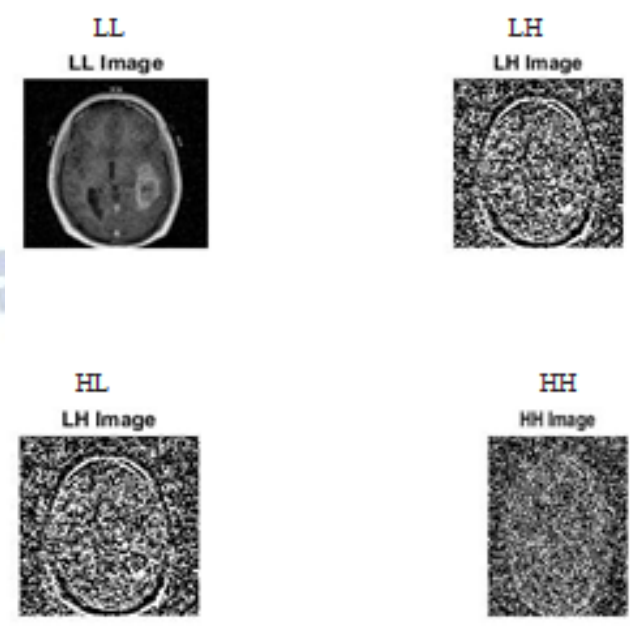

Fig 9 Decompositionlevels
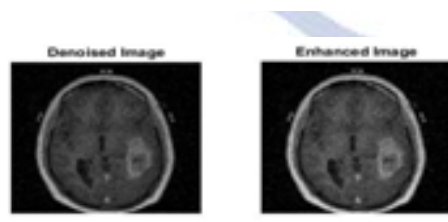

Fig.10 Denoised and the Enhanced image

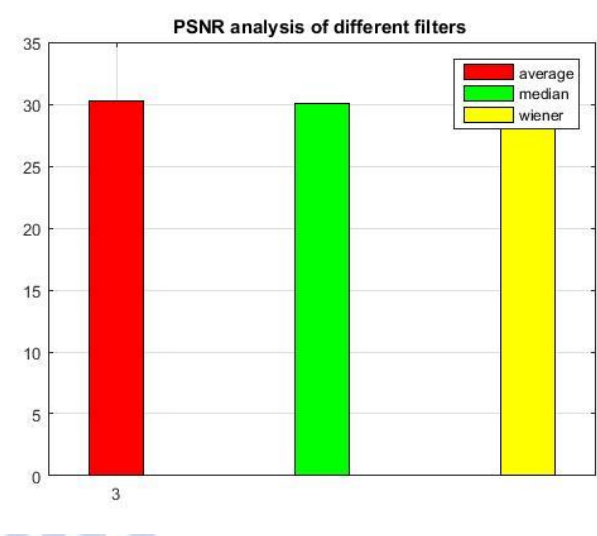

Fig. 11 PSNR Value of Denoised and Enhanced image

\section{CONCLUSION}

The MR mind image is preprocessed by denoising and goals upgrade so as to improve the nature of a image. In denoising, the clamor shows signs of improvement by wiener separating and the goals of a image is upgraded by addition based discrete wavelet change which safeguards the edges and form data. The quantitative measure shows that the goals improvement method is having better PSNR contrasted with the denoised image. In this manner, while investigating image preprocessing both the image denoising and goals improvement strategies are basic for improving the subjective execution of a image.

\section{REFERENCES}

[1] Alexandra Constantin, Ruzena Bajcsy, Berkeley Sarah,"Unsupervised Segmentation of Brain Tissue in Multivariate MRI," IEEE international conference on Biomedical imaging: from nano to Macro, pp. 89-92, April 2010.

[2] Subhranil Koley, Aurpan Majumder, "Brain MRI Segmentation for Tumor Detection using Cohesion based Self Merging Algorithm", Communication Software and Networks -ICCSN, pp. 781-785, May 2011.

[3] Suresh Kumar, Papendra Kumar, Manoj Gupta, Ashok Kumar Nagawat, "Performance Comparison of Median and wiener filter in image denosing", International Journal of Computer Applications , vol. 4, pp . 0975 - 8887, November 2010.

[4] Mona Mahmoudi, Guillermo Sapiro, "Fast Image and Video Denoising via Non-Local Means of similar neighbourhood",IEEE Signal Processing Letters , vol. 12, pp. 839-842, 2005.

[5] L. Sahawneh, B. Carroll, "Stochastic Image Denoising using Minimum Mean Squared Error (Wiener) Filtering," Electrical and Computer Engineering ,pp. 471 - 474, 2009.

[6] S. Gopinathan P. Thangavel, "A Non Linear Technique for Image Enhancement Based on Discrete Wavelet Transform", European Journal of Scientific Research, Vol. 79 , pp. 328-33, 2012.

[7] Bagawade P. Ramdas S. Bhagawat Keshav M. Patil Pradeep, "Wavelet Transform Techniques for Image Resolution Enhancement", International Journal of 
Emerging Technology and Advanced Engineering, Vol. 2, PP. 62 -65, April 2012.

[8] Syed Muhammad G. Monir, Mohammed Yakoob Siyal, Harish Kumar Maheshwari, "Noise Suppression in Functional MRI Data using Anisotropic Spatial Averaging," international conference on Information, communications and signal processing, pp. 1-4, 2009.
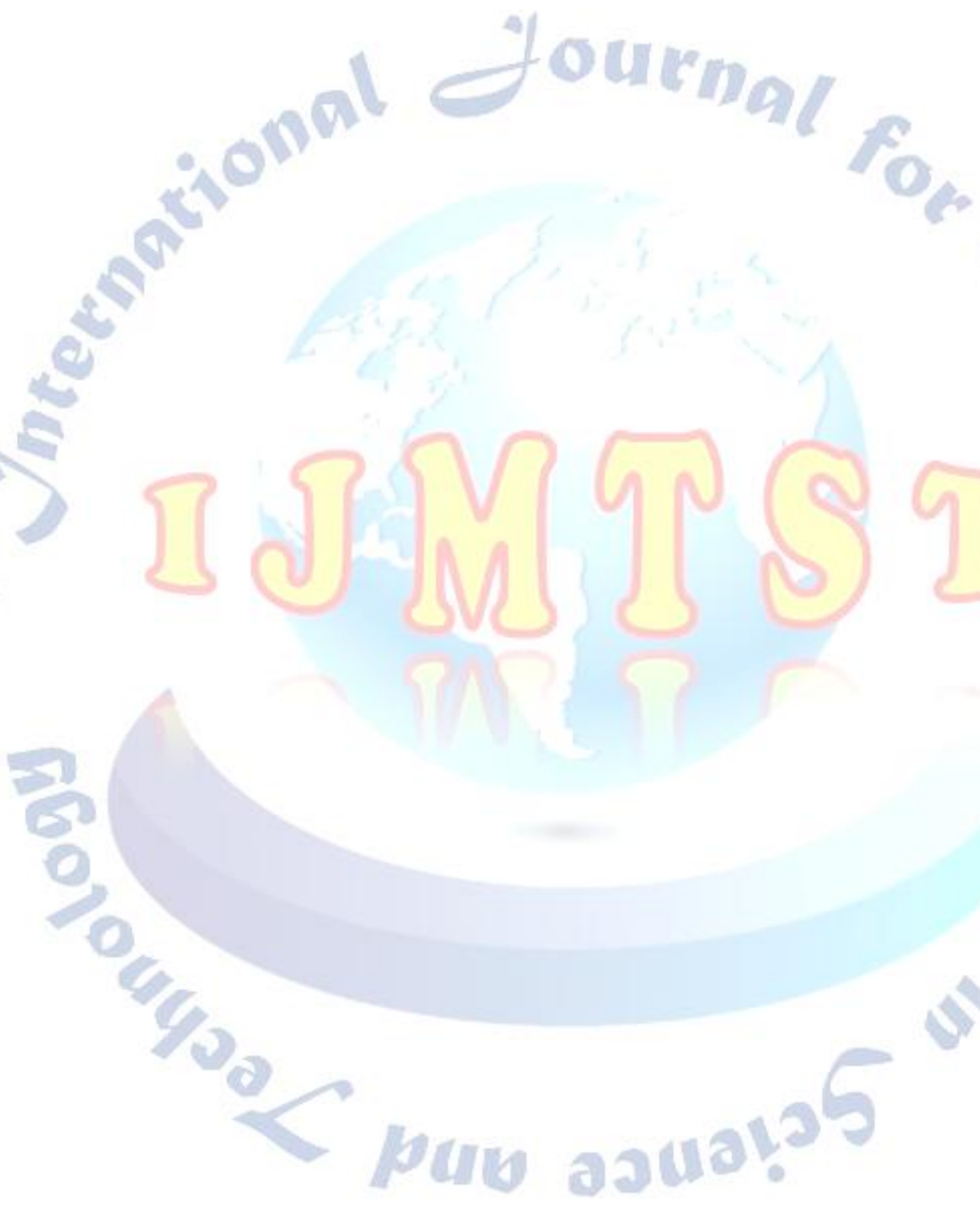